\title{
A generalization of Mehta-Wang determinant and Askey-Wilson polynomials
}

\author{
Victor J. W. Guo Masao Ishikawa $\|^{2}$ Hiroyuki Tagawa $\|^{3}$ Jiang Zeng ${ }^{4}$ \\ ${ }^{1}$ Department of Mathematics, East China Normal University, Shanghai 200062, People's Republic of China \\ ${ }^{2}$ Department of Mathematics, University of the Ryukyus, Nishihara, Okinawa 901-0213, Japan \\ ${ }^{3}$ Department of Mathematics, Wakayama University, Sakaedani, Wakayama 640-8510, Japan \\ ${ }^{4}$ Institut Camille Jordan, Université Claude Bernard Lyon 1, 69622 Villeurbanne cedex, France
}

\begin{abstract}
Motivated by the Gaussian symplectic ensemble, Mehta and Wang evaluated the $n \times n$ determinant $\operatorname{det}((a+$ $j-i) \Gamma(b+j+i))$ in 2000. When $a=0$, Ciucu and Krattenthaler computed the associated $\operatorname{Pfaffian} \operatorname{Pf}((j-i) \Gamma(b+$ $j+i)$ ) with an application to the two dimensional dimer system in 2011. Recently we have generalized the latter Pfaffian formula with a $q$-analogue by replacing the Gamma function by the moment sequence of the little $q$-Jacobi polynomials. On the other hand, Nishizawa has found a $q$-analogue of the Mehta-Wang formula. Our purpose is to generalize both the Mehta-Wang and Nishizawa formulae by using the moment sequence of the little $q$-Jacobi polynomials. It turns out that the corresponding determinant can be evaluated explicitly in terms of the Askey-Wilson polynomials.

Résumé. Motivés par des travaux sur ensemble Gaussien symplectique, Mehta et Wang sont amenés à calculer le déterminant $\operatorname{det}((a+j-i) \Gamma(b+j+i))$ de taille $n \times n$ en 2000. Lorsque $a=0$, Ciucu et Krattenthaler ont calculé le Pfaffien $\operatorname{Pf}((j-i) \Gamma(b+j+i))$ avec une application au système à deux dimènsions dimeres en 2011. Récemment nous avons généralisé le dernier Pfaffien avec un $q$-analogue en remplacant la fonction Gamma par les moments de petits $q$-polynômes de Jacobi. Par ailleurs, Nishizawa a trouvé un $q$-analogue de la formule de Mehta-Wang. Dans cet article nous démontrons une formule qui généralise à la fois la formule de Mehta-Wang et celle de Nishizawa en utilisant les moments de petits $q$-polynômes de Jacobi. Il en resulte que le determinant correspondant peut s'écrire de facon explicite à l'aide des polynômes de Askey-Wilson.
\end{abstract}

Keywords: The Mehta-Wang determinants, the moments of the little $q$-Jacobi polynomials, the Askey-Wilson polynomials.

\section{Introduction}

Motivated by the Gaussian symplectic ensemble, [13] obtain the determinant identity

$$
\operatorname{det}((a+j-i) \Gamma(b+i+j))_{0 \leq i, j \leq n-1}=D_{n} \prod_{i=0}^{n-1} i ! \Gamma(b+i)
$$

where (N.B. the binomial coefficient $\left(\begin{array}{l}n \\ k\end{array}\right)$ is missing in [13, (7)])

$$
D_{n}=\sum_{k=0}^{n}(-1)^{k}\left(\begin{array}{l}
n \\
k
\end{array}\right)\left(\frac{b-a}{2}\right)_{k}\left(\frac{a+b}{2}\right)_{n-k},
$$

\footnotetext{
${ }^{\dagger}$ Partially supported by CNRS, Institut Camille Jordan, UMR 5208.

¥Partially supported by Grant-in-Aid for Scientific Research (C) 23540017.
} 
where $(\alpha)_{n}=\frac{\Gamma(\alpha+n)}{\Gamma(\alpha)}$ is known as the rising factorial. This $D_{n}$ satisfies the three term recurrence relation

$$
D_{-1}=0, \quad D_{0}=1, \quad D_{n+1}=a D_{n}+n(b+n-1) D_{n-1},
$$

which can be considered as the recurrence relation for a special case of the Meixner-Pollaczek polynomials (see [13, 14]), and one may notice that the sequence $\{\Gamma(b+n)\}_{n \geq 0}$ of the Gamma functions in the lefthand side can be considered as the moment sequence of the Laguerre polynomials (see, for example, [7, 8, 15]). [14] obtains a $q$-analogue of (1.1), which will be stated below. In this article we replace the Gamma functions by the moments of the little $q$-Jacobi polynomials and show that we obtain a special case of the Askey-Wilson polynomials as $D_{n}$, which also generalize the two results in our previous papers [4] 5]. Before we describe our results we need more notation.

Throughout this paper we use the standard notation for $q$-series (see [3, 7, 8]):

$$
(a ; q)_{\infty}=\prod_{k=0}^{\infty}\left(1-a q^{k}\right), \quad(a ; q)_{n}=\frac{(a ; q)_{\infty}}{\left(a q^{n} ; q\right)_{\infty}}
$$

for any integer $n$. Usually $(a ; q)_{n}$ is called the $q$-shifted factorial, and we frequently use the compact notation:

$$
\left(a_{1}, a_{2}, \ldots, a_{r} ; q\right)_{n}=\left(a_{1} ; q\right)_{n}\left(a_{2} ; q\right)_{n} \cdots\left(a_{r} ; q\right)_{n} .
$$

The ${ }_{r+1} \phi_{r}$ basic hypergeometric series is defined by

$$
{ }_{r+1} \phi_{r}\left(\begin{array}{c}
a_{1}, a_{2}, \ldots, a_{r+1} \\
b_{1}, \ldots, b_{r}
\end{array} ;, z\right)=\sum_{n=0}^{\infty} \frac{\left(a_{1}, a_{2}, \ldots, a_{r+1} ; q\right)_{n}}{\left(q, b_{1}, \ldots, b_{r} ; q\right)_{n}} z^{n} .
$$

Here we also use the $q$-Gamma function

$$
\Gamma_{q}(z)=(1-q)^{1-z} \frac{(q ; q)_{\infty}}{\left(q^{z} ; q\right)_{\infty}}
$$

the $q$-integer $[n]_{q}=\frac{1-q^{n}}{1-q}$ and the $q$-factorial $[n]_{q} !=\prod_{k=1}^{n}[k]_{q}$. The Askey-Wilson polynomials $p_{n}(x)$ (see [3, 7, 8]) satisfy the well-known recurrence relation

$$
2 x p_{n}(x)=A_{n} p_{n+1}(x)+B_{n} p_{n}(x)+C_{n} p_{n-1}(x), \quad n \geq 0,
$$

with $p_{-1}(x)=0, p_{0}(x)=1$, where

$$
\begin{aligned}
A_{n} & =\frac{1-a b c d q^{n-1}}{\left(1-a b c d q^{2 n-1}\right)\left(1-a b c d q^{2 n}\right)}, \\
C_{n} & =\frac{\left(1-q^{n}\right)\left(1-a b q^{n-1}\right)\left(1-a c q^{n-1}\right)\left(1-a d q^{n-1}\right)}{\left(1-a b c d q^{2 n-2}\right)\left(1-a b c d q^{2 n-1}\right)}\left(1-b c q^{n-1}\right)\left(1-b d q^{n-1}\right)\left(1-c d q^{n-1}\right),
\end{aligned}
$$

and

$$
\begin{aligned}
B_{n}=a+a^{-1}-A_{n} a^{-1}\left(1-a b q^{n}\right)\left(1-a c q^{n}\right)\left(1-a d q^{n}\right) \\
-C_{n} a /\left(1-a b q^{n-1}\right)\left(1-a c q^{n-1}\right)\left(1-a d q^{n-1}\right) .
\end{aligned}
$$

They have the basic hypergeometric expression

$$
p_{n}(x ; a, b, c, d ; q)=\frac{(a b, a c, a d ; q)_{n}}{a^{n}}{ }_{4} \phi_{3}\left(\begin{array}{c}
q^{-n}, a b c d q^{n-1}, a e^{\imath \theta}, a e^{-\imath \theta} \\
a b, a c, a d
\end{array} ; q, q\right)
$$


with $x=\cos \theta$, where $\imath=\sqrt{-1}$. We also use the symbol

$$
\chi(A)= \begin{cases}1 & \text { if } A \text { is true } \\ 0 & \text { if } A \text { is false }\end{cases}
$$

In [4] we have proven the Hankel determinant identity

$$
\operatorname{det}\left(\frac{(a q ; q)_{i+j+r-2}}{\left(a b q^{2} ; q\right)_{i+j+r-2}}\right)_{1 \leq i, j \leq n}=a^{\frac{n(n-1)}{2}} q^{\frac{n(n-1)(2 n-1)}{6}+\frac{n(n-1) r}{2}} \prod_{k=1}^{n} \frac{(q, b q ; q)_{k-1}(a q ; q)_{k+r-1}}{\left(a b q^{2} ; q\right)_{k+n+r-2}}
$$

for a positive integer $n$. Here

$$
\mu_{n}=\frac{(a q ; q)_{n}}{\left(a b q^{2} ; q\right)_{n}} \quad(n=0,1,2, \ldots)
$$

is the moments of the little $q$-Jacobi polynomials. In our previous paper [5], we have exploited the Pfaffian identity

$$
\begin{aligned}
& \operatorname{Pf}\left(\left(q^{i-1}-q^{j-1}\right) \frac{(a q ; q)_{i+j+r-2}}{\left(a b q^{2} ; q\right)_{i+j+r-2}}\right)_{1 \leq i, j \leq 2 n} \\
& =a^{n(n-1)} q^{\frac{n(n-1)(4 n+1)}{3}+n(n-1) r} \prod_{k=1}^{n-1}(b q ; q)_{2 k} \prod_{k=1}^{n} \frac{(q ; q)_{2 k-1}(a q ; q)_{2 k+r-1}}{\left(a b q^{2} ; q\right)_{2(k+n)+r-3}}
\end{aligned}
$$

for a positive integer $n$ (see also [11, 12]).

In [14], Nishizawa has proven the $q$-analogue of the Mehta-Wang result:

$$
\begin{aligned}
\operatorname{det}( & {\left.[a+j-i]_{q} \Gamma_{q}(b+i+j)\right)_{0 \leq i, j \leq n-1} } \\
& =q^{n a+n(n-1) b / 2+n(n-1)(2 n-7) / 6} D_{n, q} \prod_{k=0}^{n-1}[k]_{q} ! \cdot \Gamma_{q}(b+k),
\end{aligned}
$$

where $D_{n, q}$ satisfies the recurrence relation

$$
D_{-1, q}=0, \quad D_{0, q}=1, \quad D_{n+1, q}=q^{-a+n}[a]_{q} D_{n, q}+q^{-a-b}[n]_{q}[b+n-1]_{q} D_{n-1, q} .
$$

Comparing this recurrence relation with the recurrence equation

$$
2 x Q_{n}(x)=Q_{n+1}(x)+(A+B) q^{n} Q_{n}(x)+\left(1-q^{n}\right)\left(1-A B q^{n-1}\right) Q_{n-1}(x)
$$

of the Al-Salam-Chihara polynomials

$$
Q_{n}(x)=Q_{n}(x ; A, B ; q)=\frac{(A B ; q)_{n}}{A^{n}}{ }_{3} \phi_{2}\left(\begin{array}{c}
q^{-n}, A e^{\imath \theta}, A e^{-\imath \theta} \\
A B, 0
\end{array} ; q ; q\right)
$$

with $x=\cos \theta$ (see [7, 8]), we may remark that $D_{n, q}$ can be considered as a special case of the Al-SalamChihara polynomials because

$$
D_{n, q}=(-\imath)^{n} q^{-\frac{a+b}{2} n}(1-q)^{-n} Q_{n}\left(0 ; q^{\frac{a+b}{2}} \imath,-q^{\frac{b-a}{2}} \imath ; q\right)
$$


By this observation, we can write $D_{n, q}$ explicitly as

$$
D_{n, q}=\frac{\left(q^{b} ; q\right)_{n}}{q^{n(a+b)}(q-1)^{n}} \sum_{k=0}^{n} q^{k} \frac{\left(q^{-n} ; q\right)_{k}}{(q ; q)_{k}} \prod_{j=0}^{k-1} \frac{1-q^{a+b+2 j}}{1-q^{b+j}} .
$$

One natural question we may ask is what can we obtain if we replace the $q$-Gamma function in the determinant of $(1.9]$ by the moment of the little $q$-Jacobi polynomials. The aim of this paper is to answer this question, and we can express the determinant by the Askey-Wilson polynomials.

Theorem 1.1 Let $a, b$ and $c$ be parameters, and let $n \geq 1$ and $r$ be integers. Then we have

$$
\begin{aligned}
& \operatorname{det}\left(\left(q^{i-1}-c q^{j-1}\right) \frac{(a q ; q)_{i+j+r-2}}{\left(a b q^{2} ; q\right)_{i+j+r-2}}\right)_{1 \leq i, j \leq n} \\
& =(-1)^{n} a^{\frac{n(n-3)}{2}} q^{\frac{n(n+1)(2 n-5)}{6}}+\frac{n(n-3) r}{2}\left(a b c q^{r+1} ; q^{2}\right)_{n} \prod_{k=1}^{n} \frac{(q ; q)_{k-1}(a q ; q)_{k+r}(b q ; q)_{k-2}}{\left(a b q^{2} ; q\right)_{k+n+r-2}} \\
& \times_{4} \phi_{3}\left(\begin{array}{l}
q^{-n}, a^{\frac{1}{2}} c^{\frac{1}{2}} q^{\frac{r+1}{2}},-a^{\frac{1}{2}} c^{\frac{1}{2}} q^{\frac{r+1}{2}}, a b q^{n+r} \\
a q^{r+1}, a^{\frac{1}{2}} b^{\frac{1}{2}} c^{\frac{1}{2}} q^{\frac{r+1}{2}},-a^{\frac{1}{2}} b^{\frac{1}{2}} c^{\frac{1}{2}} q^{\frac{r+1}{2}} ; q, q
\end{array}\right) \\
& =(-\imath)^{n} a^{\frac{n(n-2)}{2}} c^{\frac{n}{2}} q^{\frac{n(n-2)(2 n+1)}{6}+\frac{n(n-2) r}{2}} \prod_{k=1}^{n} \frac{(q ; q)_{k-1}(a q ; q)_{k+r-1}(b q ; q)_{k-2}}{\left(a b q^{2} ; q\right)_{k+n+r-2}} \\
& \times p_{n}\left(0 ; a^{\frac{1}{2}} c^{\frac{1}{2}} q^{\frac{r+1}{2}} \imath,-a^{\frac{1}{2}} c^{-\frac{1}{2}} q^{\frac{r+1}{2}} \imath, b^{\frac{1}{2}} \imath,-b^{\frac{1}{2}} \imath ; q\right) .
\end{aligned}
$$

Remark 1.2 If we put $c=0$ in (1.15), then we recover our previous result (1.7) easily by using the $q$-Chu-Vandermonde formula [3] (1.5.3)]

$$
{ }_{2} \phi_{1}\left(\begin{array}{c}
a, q^{-n} \\
c
\end{array} ; q, q\right)=\frac{(c / a ; q)_{n}}{(c ; q)_{n}} a^{n} .
$$

If we put $a=q^{\alpha-1}, b=0, c=q^{\gamma}$ and $r=0$ in 1.15, then the left-hand side equals

$$
\frac{q^{\frac{n(n-1)}{2}}(1-q)^{n^{2}}}{\left\{\Gamma_{q}(\alpha)\right\}^{n}} \operatorname{det}\left([\gamma+j-i]_{q} \Gamma_{q}(\alpha+i+j-2)\right)_{1 \leq i, j \leq n}
$$

because of $\left(q^{\alpha} ; q\right)_{n}=(1-q)^{n} \cdot \frac{\Gamma_{q}(\alpha+n)}{\Gamma_{q}(\alpha)}$, and the right-hand side equals

$$
(-\imath)^{n} q^{\frac{n(n-2)}{2} \alpha+\frac{n}{2} \gamma+\frac{n(n-1)(n-2)}{3}} \prod_{k=1}^{n}(q ; q)_{k-1}\left(q^{\alpha} ; q\right)_{k-1} \cdot Q_{n}\left(0 ; q^{\frac{\alpha+\gamma}{2}} \imath,-q^{\frac{\alpha-\gamma}{2}} \imath ; q\right)
$$

because of the relation $Q_{n}(x ; A, B ; q)=p_{n}(x ; A, B, 0,0 ; q)$ between the Al-Salam-Chihara polynomials and the Askey-Wilson polynomials. Hence we obtain Nishizawa's formula (1.9) as a corollary.

Corollary 1.3 Let $a, b$ and $c$ be parameters, and let $n \geq 1$ and $r$ be integers.

(i) If the size $n=2 m$ of the matrix is even, then we have

$$
\operatorname{det}\left(\left(q^{i-1}-c q^{j-1}\right) \frac{(a q ; q)_{i+j+r-2}}{\left(a b q^{2} ; q\right)_{i+j+r-2}}\right)_{1 \leq i, j \leq 2 m}
$$




$$
\begin{aligned}
& =a^{2 m(m-1)} c^{m} q^{\frac{2 m(m-1)(4 m+1)}{3}+2 m(m-1) r} \prod_{k=1}^{m}\left\{\frac{(q ; q)_{2 k-1}(a q ; q)_{2 k+r-1}(b q ; q)_{2 k-2}}{\left(a b q^{2} ; q\right)_{2(k+m)+r-3}}\right\}^{2} \\
& \times{ }_{4} \phi_{3}\left(\begin{array}{c}
q^{-2 m}, b^{-1} q^{-2 m+1}, c, c^{-1} \\
q, a q^{r+1}, a^{-1} b^{-1} q^{1-4 m-r}
\end{array} q^{2}, q^{2}\right) \\
& =(-1)^{m} a^{m(2 m-1)} b^{m} c^{m} q^{\frac{m\left(8 m^{2}+3 m-2\right)}{3}+m(2 m-1) r} \prod_{k=1}^{2 m} \frac{(q ; q)_{k-1}(a q ; q)_{k+r-1}}{\left(a b q^{2} ; q\right)_{k+2 m+r-2}} \\
& \times \prod_{k=1}^{m}\left\{(b q ; q)_{2 k-2}\right\}^{2} \cdot p_{m}\left(\frac{c+c^{-1}}{2} ; 1, q, a q^{r+1}, a^{-1} b^{-1} q^{1-4 m-r} ; q^{2}\right) .
\end{aligned}
$$

(ii) If the size $n=2 m+1$ of the matrix is odd, then we have

$$
\begin{aligned}
& \operatorname{det}\left(\left(q^{i-1}-c q^{j-1}\right) \frac{(a q ; q)_{i+j+r-2}}{\left(a b q^{2} ; q\right)_{i+j+r-2}}\right)_{1 \leq i, j \leq 2 m+1} \\
& =a^{2 m^{2}} c^{m} q^{\frac{2 m(m+1)(4 m-1)}{3}+2 m^{2} r} \cdot \frac{1-c}{1-q} \cdot \prod_{k=1}^{m+1} \frac{(q ; q)_{2 k-1}(a q ; q)_{2 k+r-2}(b q ; q)_{2 k-2}}{\left(a b q^{2} ; q\right)_{2(k+m-1)+r}} \\
& \times \prod_{k=1}^{m} \frac{(q ; q)_{2 k-1}(a q ; q)_{2 k+r}(b q ; q)_{2 k-2}}{\left(a b q^{2} ; q\right)_{2(k+m-1)+r}} \cdot{ }_{4} \phi_{3}\left(\begin{array}{c}
q^{-2 m}, b^{-1} q^{-2 m+1}, c q, c^{-1} q \\
q^{3}, a q^{r+2}, a^{-1} b^{-1} q^{-4 m-r} ; q^{2}, q^{2}
\end{array}\right)
\end{aligned}
$$

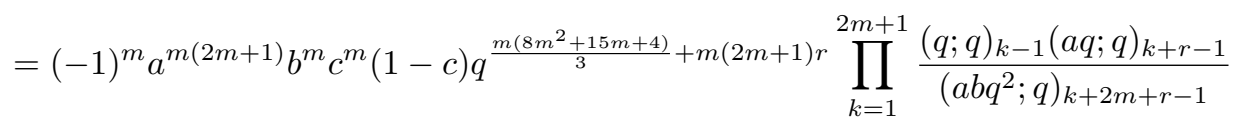

$$
\begin{aligned}
& \times \prod_{k=1}^{m+1}(b q ; q)_{2 k-2} \cdot \prod_{k=1}^{m}(b q ; q)_{2 k-2} \cdot p_{m}\left(\frac{c+c^{-1}}{2} ; q, q^{2}, a q^{r+1}, a^{-1} b^{-1} q^{-4 m-r-1} ; q^{2}\right) \text {. }
\end{aligned}
$$

Remark 1.4 If we put $c=1$ in (1.18) for the even case, then it is clear that the ${ }_{4} \phi_{3}$ sum reduces to 1 , so that the determinant becomes the product which equals the square of the Pfaffian (1.8) obtained in [5]. Meanwhile, it does not suffice to prove (1.8) since it is not so trivial to take the square root of the determinant and determine the sign (see [2] 57). If we put $c=1$ in (1.20) for the odd case, then the factor $(1-c)$ reduces the right-hand side to 0.

\section{Determinant formula for arbitrary rows}

In our previous paper [4], we prove the following formula in which the rows are arbitrary chosen. Let $n$ be a positive integer, and $k_{1}, \ldots, k_{n}$ be arbitrary positive integers. Then we have

$$
\begin{aligned}
& \operatorname{det}\left(\frac{(a q ; q)_{k_{i}+j-2}}{\left(a b q^{2} ; q\right)_{k_{i}+j-2}}\right)_{1 \leq i, j \leq n}=a^{\frac{n(n-1)}{2}} q^{\frac{(n+1) n(n-1)}{6}} \\
& \times \prod_{i=1}^{n} \frac{(a q ; q)_{k_{i}-1}}{\left(a b q^{2} ; q\right)_{k_{i}+n-2}} \prod_{1 \leq i<j \leq n}\left(q^{k_{i}-1}-q^{k_{j}-1}\right) \prod_{j=1}^{n}(b q ; q)_{j-1} .
\end{aligned}
$$

This formula is a generalization of (1.7) and a special case is obtained in [10, Theorem 3]. In this section we give this type formula, i.e., Theorem 2.1. which is crucial to prove Theorem 1.1 . 
First we fix some notation. If $a$ and $b$ are integers, we write $[a, b]=\{x \in \mathbb{Z} \mid a \leq x \leq b\}$. We also write $[n]=[1, n]$ for short. If $S$ is a finite set and $r$ a nonnegative integer, let $\left(\begin{array}{l}S \\ r\end{array}\right)$ denote the set of all $r$-element subsets of $S$. Let $A$ be an $m \times n$ matrix. If $\mathbf{i}=\left(i_{1}, \ldots, i_{r}\right)$ is an $r$-tuple of positive integers and $\mathbf{j}=\left(j_{1}, \ldots, j_{s}\right)$ is an $s$-tuple of positive integers, then let $A_{\mathbf{j}}^{\mathbf{i}}=A_{j_{1}, \ldots, j_{s}}^{i_{1}, \ldots, i_{r}}$ denote the submatrix formed by selecting the row $\mathbf{i}$ and the column $\mathbf{j}$ from $A$. Then the following theorem generalize (2.1).

Theorem 2.1 Let $a, b$ and $c$ be parameters. Let $n$ be a positive integer, and $\mathbf{k}=\left(k_{1}, \ldots, k_{n}\right)$ be an $n$-tuple of positive integers. Then we have

$$
\begin{aligned}
\operatorname{det} & \left(\left(q^{k_{i}-1}-c q^{j-1}\right) \frac{(a q ; q)_{k_{i}+j-2}}{\left(a b q^{2} ; q\right)_{k_{i}+j-2}}\right)_{1 \leq i, j \leq n} \\
= & a^{\frac{n(n-3)}{2}} q^{\frac{n(n+1)(n-4)}{6}} \prod_{i=1}^{n} \frac{(a q ; q)_{k_{i}-1}(b q ; q)_{i-2}}{\left(a b q^{2} ; q\right)_{k_{i}+n-2}} \prod_{1 \leq i<j \leq n}\left(q^{k_{i}-1}-q^{k_{j}-1}\right) \\
& \times \sum_{\nu=0}^{n}(-1)^{n-\nu}\left(a b c q^{2 \nu+1} ; q^{2}\right)_{n-\nu}\left(a c q ; q^{2}\right)_{\nu} R_{n, \nu}(\mathbf{k}, a, b ; q)
\end{aligned}
$$

where

$$
R_{n, \nu}(\mathbf{k}, a, b ; q)=\sum_{(\mathbf{i}, \mathbf{j})} q^{\sum_{l=1}^{n-\nu} i_{l}-n+\nu} \prod_{l=1}^{n-\nu}\left(1-a q^{k_{i_{l}}-i_{l}+l+\nu}\right) \prod_{l=1}^{\nu}\left(1-a b q^{k_{j_{l}}+j_{l}-l+\nu-1}\right) .
$$

Here the sum on the right-hand side runs over all pairs $(\mathbf{i}, \mathbf{j})$ such that $[n]$ is a disjoint union of $\mathbf{i}=$ $\left\{i_{1}, \ldots, i_{n-\nu}\right\} \in\left(\begin{array}{c}{[n]} \\ n-\nu\end{array}\right)$ and $\mathbf{j}=\left\{j_{1}, \ldots, j_{\nu}\right\} \in\left(\begin{array}{c}{[n]} \\ \nu\end{array}\right)(i . e ., \mathbf{i} \cup \mathbf{j}=[n]$ and $\mathbf{i} \cap \mathbf{j}=\emptyset)$.

For example, if $n=3$ and $\nu=2$, then the pairs $(\mathbf{i}, \mathbf{j})$ runs over

$$
\{(\{1\},\{2,3\}),(\{2\},\{1,3\}),(\{3\},\{1,2\})\} .
$$

Hence we have

$$
\begin{aligned}
R_{3,2}\left(\left\{k_{1}, k_{2}, k_{3}\right\}\right. & , a, b ; q)=\left(1-a q^{k_{1}+2}\right)\left(1-a b q^{k_{2}+2}\right)\left(1-a b q^{k_{3}+2}\right) \\
& +q\left(1-a q^{k_{2}+1}\right)\left(1-a b q^{k_{1}+1}\right)\left(1-a b q^{k_{3}+2}\right) \\
& +q^{2}\left(1-a q^{k_{3}}\right)\left(1-a b q^{k_{1}+1}\right)\left(1-a b q^{k_{2}+1}\right)
\end{aligned}
$$

Here we have no space to describe the proof of Theorem 2.1. We need some intensive use of linear algebra for the proof. The interested reader should consult [6]. Here we describe only the sketch of the proof.

Let $n$ be a positive integer, and let $a, b, c$ and $q$ be parameters. For an index set $\mathbf{k}=\left\{k_{1}, \ldots, k_{n}\right\}$ of positive integers, let $M_{n}(\mathbf{k}, a, b, c ; q)=\left(M_{n}(\mathbf{k}, a, b, c ; q)_{i, j}\right)_{1 \leq i, j \leq n}$ denote the matrix whose $(i, j)$ entry is given by

$$
M_{n}(\mathbf{k}, a, b, c ; q)_{i, j}=\left(q^{k_{i}-1}-c q^{j-1}\right)\left(a q^{k_{i}} ; q\right)_{j-1}\left(a b q^{k_{i}+j} ; q\right)_{n-j} .
$$

Then we have

$$
\operatorname{det}\left(\left(q^{k_{i}-1}-c q^{j-1}\right) \frac{(a q ; q)_{k_{i}+j-2}}{\left(a b q^{2} ; q\right)_{k_{i}+j-2}}\right)_{1 \leq i, j \leq n}=\prod_{i=1}^{n} \frac{(a q ; q)_{k_{i}-1}}{\left(a b q^{2} ; q\right)_{k_{i}+n-2}} \cdot \operatorname{det} M_{n}(\mathbf{k}, a, b, c ; q) .
$$


Hence it is enough to evaluate $\operatorname{det} M_{n}(\mathbf{k}, a, b, c ; q)$ to prove Theorem 2.1. The main task of this evaluation is to show the following recurrence equation:

$$
\begin{aligned}
& \frac{\operatorname{det} M_{n}(\mathbf{k}, a, b, c ; q)}{a^{n-2}(b q ; q)_{n-2} \prod_{i=1}^{n-1}\left(q^{k_{i}}-q^{k_{n}}\right)} \\
& =q^{-1}(1-a c q)\left(1-a b q^{k_{n}+n-1}\right) \operatorname{det} M_{n-1}\left(\mathbf{k}^{\prime}, a q, b, c q ; q\right) \\
& \quad-q^{n(n-3) / 2}\left(1-a b c q^{2 n-1}\right)\left(1-a q^{k_{n}}\right) \operatorname{det} M_{n-1}\left(\mathbf{k}^{\prime}, a, b, c ; q\right),
\end{aligned}
$$

where $\mathbf{k}^{\prime}=\left\{k_{1}, \ldots, k_{n-1}\right\}$ denote the subset of the first $(n-1)$ indices of $\mathbf{k}=\left\{k_{1}, \ldots, k_{n-1}, k_{n}\right\}$. This identity enable us to prove 2.2 by induction.

We introduce four triangular matrices $X_{n}(\mathbf{k}, a ; q), Y_{n}(q), L_{n}(\mathbf{k}, a, b ; q)$ and $U_{n}(q)$ which play an important role to manipulate $M_{n}(\mathbf{k}, a, b, c ; q)$ in 2.5 . Let $X_{n}(\mathbf{k}, a ; q)=\left(X(\mathbf{k}, a ; q)_{i, j}\right)_{1 \leq i, j \leq n}$ and $Y_{n}(q)=\left(Y_{n}(q)_{i, j}\right)_{1 \leq i, j \leq n}$ be the $n \times n$ lower triangular matrices whose $(i, j)$-entry is, respectively, given by

$$
\begin{aligned}
& X(\mathbf{k}, a ; q)_{i, j}=-\frac{\chi(i \geq j)}{q^{k_{j}}\left(1-a q^{k_{j}}\right) \prod_{\substack{l=1 \\
l \neq j}}^{i}\left(q^{k_{l}}-q^{k_{j}}\right)}, \\
& Y_{n}(q)_{i, j}=(-1)^{i+j} q^{-\frac{(i-j)(2 n+1-i-j)}{2}}\left[\begin{array}{c}
n-j \\
i-j
\end{array}\right]_{q} .
\end{aligned}
$$

Similarly, let $L_{n}(\mathbf{k}, a, b ; q)=\left(L_{n}(\mathbf{k}, a, b ; q)_{i, j}\right)_{1 \leq i, j \leq n}\left(\operatorname{resp} . U_{n}(q)=\left(U(q)_{i, j}\right)_{1 \leq i, j \leq n}\right)$ be the $n \times n$ lower (resp. upper) triangular matrix whose $(i, j)$-entry is, respectively, given by

$$
\begin{aligned}
& L_{n}(\mathbf{k}, a, b ; q)_{i, j}=-\frac{\chi(i \geq j)}{q^{k_{j}}\left(1-a b q^{k_{j}+n-1}\right) \prod_{\substack{l=1 \\
l \neq j}}^{i}\left(q^{k_{l}}-q^{k_{j}}\right)}, \\
& U(q)_{i, j}=(-1)^{i+j} q^{\frac{(j-i)(j-i+1)}{2}}\left[\begin{array}{c}
j-1 \\
j-i
\end{array}\right]_{q} .
\end{aligned}
$$

We define the $n \times n$ matrices $P_{n}(\mathbf{k}, a, b, c ; q)$ and $Q_{n}(\mathbf{k}, a, b, c ; q)$ by

$$
\begin{aligned}
& P_{n}(\mathbf{k}, a, b, c ; q)=X_{n}(\mathbf{k}, a ; q) M_{n}(\mathbf{k}, a, b, c ; q) Y_{n}(q) \\
& Q_{n}(\mathbf{k}, a, b, c ; q)=L_{n}(\mathbf{k}, a, b ; q) M_{n}(\mathbf{k}, a, b, c ; q) U_{n}(q)
\end{aligned}
$$

Since $X_{n}(\mathbf{k}, a ; q), L_{n}(\mathbf{k}, a, b ; q)$ are triangular and $Y_{n}(q), U_{n}(q)$ are unitriangular, we easily obtain

$$
\begin{aligned}
& \operatorname{det} P_{n}(\mathbf{k}, a, b, c ; q)=\frac{(-1)^{n} \operatorname{det} M_{n}(\mathbf{k}, a, b, c ; q)}{q^{\sum_{i=1}^{n} k_{i}} \prod_{i=1}^{n}\left(1-a q^{k_{i}}\right) \prod_{1 \leq i<j \leq n}\left(q^{k_{i}}-q^{k_{j}}\right)}, \\
& \operatorname{det} Q_{n}(\mathbf{k}, a, b, c ; q)=\frac{(-1)^{n} \operatorname{det} M_{n}(\mathbf{k}, a, b, c ; q)}{q^{\sum_{i=1}^{n} k_{i}} \prod_{i=1}^{n}\left(1-a b q^{k_{i}+n-1}\right) \prod_{1 \leq i<j \leq n}\left(q^{k_{i}}-q^{k_{j}}\right)} .
\end{aligned}
$$

The key to prove 2.6$]$ is the following lemma:

Lemma 2.2 Let $n$ be a positive integer, and let $a, b, c$ and $q$ be parameters. Let $P_{n}(\mathbf{k}, a, b, c ; q)$ and $Q_{n}(\mathbf{k}, a, b, c ; q)$ be as defined above. When $\mathbf{k}=\left\{k_{1}, \ldots, k_{n-1}, k_{n}\right\}$ is a row index set, let $\mathbf{k}^{\prime}=$ 
$\left\{k_{1}, \ldots, k_{n-1}\right\}$ denote the subset of the first $(n-1)$ indices of $\mathbf{k}$. Then we have

$$
\begin{gathered}
\operatorname{det} P_{n}(\mathbf{k}, a, b, c ; q)_{[2, n]}^{[1, n-1]}=\frac{(-1)^{n-1} \operatorname{det} M_{n-1}\left(\mathbf{k}^{\prime}, a q, b, c q ; q\right)}{q^{\sum_{i=1}^{n-1} k_{i}} \prod_{1 \leq i<j<n}\left(q^{k_{i}}-q^{k_{j}}\right)}, \\
\operatorname{det} Q_{n}(\mathbf{k}, a, b, c ; q)_{[1, n-1]}^{[1, n-1]}=\frac{(-1)^{n-1} \operatorname{det} M_{n-1}\left(\mathbf{k}^{\prime}, a, b, c ; q\right)}{q^{\sum_{i=1}^{n-1} k_{i}} \prod_{1 \leq i<j<n}\left(q^{k_{i}}-q^{k_{j}}\right)}, \\
\frac{\operatorname{det} P_{n}(\mathbf{k}, a, b, c ; q)_{[1, n-1]}^{[1, n-1]}}{\prod_{\nu=1}^{n-1}\left(1-a b q^{k_{\nu}+n-1}\right)}=(-q)^{-n+1} \frac{\operatorname{det} Q_{n}(\mathbf{k}, a, b, c ; q)_{[2, n]}^{[1, n-1]}}{\prod_{\nu=1}^{n-1}\left(1-a q^{k_{\nu}}\right)}
\end{gathered}
$$

Now we are in position to prove Theorem 2.1. In fact the proof is straightforward by induction.

Proof of Theorem 2.1. First, we note that, for any integers $n$ and $\nu$, it holds

$$
\begin{array}{r}
R_{n, \nu}(\mathbf{k}, a, b ; q)=\left(1-a b q^{k_{n}+n-1}\right) R_{n-1, \nu-1}\left(\mathbf{k}^{\prime}, a q, b ; q\right) \\
+q^{n-1}\left(1-a q^{k_{n}}\right) R_{n-1, \nu}\left(\mathbf{k}^{\prime}, a, b ; q\right),
\end{array}
$$

where $\mathbf{k}=\left\{k_{1}, \ldots, k_{n-1}, k_{n}\right\}$ and $\mathbf{k}^{\prime}=\left\{k_{1}, \ldots, k_{n-1}\right\}$ are as before. 2.16 follows from the definition 2.3 of $R_{n, \nu}(\mathbf{k}, a, b ; q)$ by considering two exclusive cases, $j_{\nu}=n$ or $i_{n-\nu}=n$. Now we prove the identity

$$
\begin{aligned}
& \operatorname{det} M_{n}(\mathbf{k}, a, b, c ; q)=(-1)^{n} a^{\frac{n(n-3)}{2}} q^{\frac{n(n+1)(n-4)}{6}} \prod_{i=1}^{n}(b q ; q)_{i-2} \\
& \quad \times \prod_{1 \leq i<j \leq n}\left(q^{k_{i}-1}-q^{k_{j}-1}\right) \sum_{\nu=0}^{n}(-1)^{\nu}\left(a b c q^{2 \nu+1} ; q^{2}\right)_{n-\nu}\left(a c q ; q^{2}\right)_{\nu} R_{n, \nu}(\mathbf{k}, a, b ; q),
\end{aligned}
$$

by induction on $n$. If $n=1$, then the left-hand side of 2.17) is trivially $q^{k_{1}-1}-c$ from 2.4. It is straightforward computation to check the right-hand side equals $q^{k_{1}-1}-c$. Assume $n>1$ and 2.17) holds up to $(n-1)$. Using 2.6 and the induction hypothesis, we obtain

$$
\begin{aligned}
& \frac{\operatorname{det} M_{n}(\mathbf{k}, a, b, c ; q)}{(-1)^{n} a^{\frac{n(n-3)}{2}} q^{\frac{n\left(n^{2}-6 n-1\right)}{6}} \prod_{i=1}^{n}(b q ; q)_{i-2} \prod_{1 \leq i<j \leq n}\left(q^{k_{i}}-q^{k_{j}}\right)} \\
& =\left(1-a b q^{k_{n}+n-1}\right) \sum_{\nu=0}^{n-1}(-1)^{\nu+1}\left(a b c q^{2 \nu+3} ; q^{2}\right)_{n-\nu-1}\left(a c q ; q^{2}\right)_{\nu+1} R_{n-1, \nu}\left(\mathbf{k}^{\prime}, a q, b ; q\right) \\
& +q^{n-1}\left(1-a q^{k_{n}}\right) \sum_{\nu=0}^{n-1}(-1)^{\nu}\left(a b c q^{2 \nu+1} ; q^{2}\right)_{n-\nu}\left(a c q ; q^{2}\right)_{\nu} R_{n-1, \nu}\left(\mathbf{k}^{\prime}, a, b ; q\right) .
\end{aligned}
$$

Replacing $\nu+1$ by $\nu$ in the first sum and applying 2.16, we establish 2.17) for $n$. Hence 2.17) holds for an arbitrary positive integer $n$. Finally, 2.5) and 2.17] immediately implies (2.2). This completes the proof of Theorem 2.1

For the detail of the proofs of the lemmas in this section, the reader can consult [6]

\section{Proof of the main theorems}

The aim of this section is to describe the outline of the proofs of the theorems in Section 1 i.e., Theorem 1.1 from Theorem 2.1 and then prove Corollary 1.3 from Theorem 1.1. Once we prove Theorem 2.1. 
then it is easy and straightforward to prove the main theorems mainly by induction. In fact, to prove Theorem 1.1. we need to set $\mathbf{k}=[n]=\{1,2, \ldots, n\}$ in 2.2. Hence, the following lemma is essential to prove (1.16.

Lemma 3.1 If we put $\mathbf{k}=[n]$ in $[2.2$, then we obtain

$$
R_{n, \nu}([n], a, b ; q)=q^{\frac{(n-\nu)(n-\nu-1)}{2}}\left[\begin{array}{l}
n \\
\nu
\end{array}\right]_{q}\left(a q^{\nu+1} ; q\right)_{n-\nu}\left(a b q^{n} ; q\right)_{\nu} .
$$

In fact the proof of Theorem 1.1 is quite straightforward by substitution $\mathbf{k}=[n]$ into 2.2 using 3.1. We use some well-known $q$-series identities. The details are described in [6].

To derive Corollary 1.3 from Theorem 1.1 the following proposition plays a crucial role:

Proposition 3.2 Let $n$ be an integer, $a, b$ and $c$ be arbitrary parameters. Then we have

$$
\begin{aligned}
p_{n}(0 ; a, b, c,-c ; q)= & (-1)^{m} a^{m} b^{m} c^{2 m} q^{m(3 m-1)}\left(-c^{2} ; q^{2}\right)_{m} \\
& \times p_{m}\left(x_{0} ; 1, q, a b,-a^{-1} b^{-1} c^{-2} q^{-4 m+2} ; q^{2}\right),
\end{aligned}
$$

if $n=2 m$ is even, and

$$
\begin{gathered}
p_{n}(0 ; a, b, c,-c ; q)=(-1)^{m+1} a^{m} b^{m+1} c^{2 m}\left(1+a b^{-1}\right) q^{m(3 m+1)}\left(-c^{2} ; q^{2}\right)_{m+1} \\
\times p_{m}\left(x_{0} ; q, q^{2}, a b,-a^{-1} b^{-1} c^{-2} q^{-4 m} ; q^{2}\right)
\end{gathered}
$$

if $n=2 m+1$ is odd, where $x_{0}=-\frac{a b^{-1}+a^{-1} b}{2}$.

When $b=-a$, replacing $c$ by $b$, one gets incidentally the following known result due to Andrews (see [3, (II.17)]).

Corollary 3.3

$$
p_{n}(0 ; a,-a, b,-b ; q)= \begin{cases}(-1)^{m}\left(q,-a^{2},-b^{2}, a^{2} b^{2} q^{2 m} ; q^{2}\right)_{m} & \text { if } n=2 m, \\ 0 & \text { if } n=2 m+1 .\end{cases}
$$

To prove of Proposition 3.2, we use the following contiguous relations for ${ }_{4} \phi_{3}$.

Proposition 3.4 Let $z, a, b, c, d, e, f, g$ and $q$ be arbitrary parameters. Then we have

$$
\begin{aligned}
{ }_{4} \phi_{3} & \left(\begin{array}{c}
a, b q, c, d \\
e, f, g
\end{array} ; q, z\right)-{ }_{4} \phi_{3}\left(\begin{array}{c}
a q, b, c, d \\
e, f, g
\end{array} ; q, z\right) \\
& =\frac{z(b-a)(1-c)(1-d)}{(1-e)(1-f)(1-g)}{ }_{4} \phi_{3}\left(\begin{array}{c}
a q, b q, c q, d q \\
e q, f q, g q
\end{array} ; q, z\right) \\
(1-f)(a-e){ }_{4} \phi_{3}\left(\begin{array}{c}
a, b, c, d \\
e q, f, g
\end{array} ; q, z\right)-(1-e)(a-f){ }_{4} \phi_{3}\left(\begin{array}{c}
a, b, c, d \\
e, f q, g
\end{array} ; q, z\right) & \\
= & (1-a)(f-e){ }_{4} \phi_{3}\left(\begin{array}{c}
a q, b, c, d \\
e q, f q, g
\end{array} ; q, z\right)
\end{aligned}
$$

and

$$
\begin{aligned}
& (1-e)(1-f)(1-g)_{4} \phi_{3}\left(\begin{array}{c}
a, b, c, d \\
e, f, g
\end{array} ; q, q\right) \\
& \quad=c(1-e)\left(1-\frac{f}{c}\right)\left(1-\frac{g}{c}\right){ }_{4} \phi_{3}\left(\begin{array}{c}
a q, b q, c, d \\
e, f q, g q
\end{array} ; q, q\right) \\
& \quad+d(1-c)\left(1-\frac{e}{d}\right)\left(1-\frac{f g}{c d}\right){ }_{4} \phi_{3}\left(\begin{array}{c}
a q, b q, c q, d \\
e q, f q, g q
\end{array} ; q, q\right)
\end{aligned}
$$


where, in the last identity, we assume abcdq $=$ efg and $a=q^{-n}$ for some nonnegative integer $n$.

Remark 3.5 The contiguous relations 3.5) (resp. 3.6) correspond to (3.2) (resp. (3.10)) in [9], meanwhile (3.6) can be written as a contiguous relation for ${ }_{8} W_{7}$. In fact, if one uses Watson's transformation formula [3] (2.5.1)]

$$
{ }_{8} W_{7}\left(a ; b, c, d, e, q^{-n} ; q, \frac{a^{2} q^{n+2}}{b c d e}\right)=\frac{\left(a q, \frac{a q}{d e} ; q\right)_{n}}{\left(\frac{a q}{d}, \frac{a q}{e} ; q\right)_{n}} 4 \phi_{3}\left(\begin{array}{c}
q^{-n}, d, e, \frac{a q}{b c} \\
\frac{a q}{b}, \frac{a q}{c}, \frac{d e q-n}{a}
\end{array} ;, q\right)
$$

for a terminating very-well-poised ${ }_{8} \phi_{7}$ series, where

$$
{ }_{r+1} W_{r}\left(a_{1} ; a_{4}, \ldots, a_{r+1} ; q, z\right)={ }_{r+1} \phi_{r}\left(\begin{array}{c}
a_{1}, q a_{1}^{\frac{1}{2}},-q a_{1}^{\frac{1}{2}}, a_{4}, \ldots, a_{r+1} \\
a_{1}^{\frac{1}{2}},-a_{1}^{\frac{1}{2}}, \frac{q a_{1}}{a_{4}}, \ldots, \frac{q a_{1}}{a_{r+1}}
\end{array} ; q, z\right),
$$

then (3.7) is equivalent to

$$
\begin{aligned}
& (c-a)(d-a q)(e-a q)\left(b-a q^{n}\right){ }_{8} W_{7}\left(a ; b, c q, d, e, q^{-n} ; q, \frac{a^{2} q^{n+1}}{b c d e}\right) \\
= & a(1-b)(1-a q)(d e-a q)\left(1-c q^{n}\right){ }_{8} W_{7}\left(a q ; b q, c q, d, e, q^{-n+1} ; q, \frac{a^{2} q^{n+1}}{b c d e}\right) \\
+ & (b c-a)(d-a q)(e-a q)\left(1-a q^{n}\right){ }_{8} W_{7}\left(a ; b, c, d, e, q^{-n+1} ; q, \frac{a^{2} q^{n+1}}{b c d e}\right) .
\end{aligned}
$$

\section{A quadratic relation}

First we recall the reader a well-known theorem for determinants. The following identity is known as the Desnanot-Jacobi adjoint matrix theorem [1, Theorem 3.12]

$$
\operatorname{det} A_{[2, n-1]}^{[2, n-1]} \operatorname{det} A_{[n]}^{[n]}=\operatorname{det} A_{[n-1]}^{[n-1]} \operatorname{det} A_{[2, n]}^{[2, n]}-\operatorname{det} A_{[2, n]}^{[n-1]} \operatorname{det} A_{[n-1]}^{[2, n]} .
$$

Let

$$
D_{n}(a, b, c ; q)=\operatorname{det}\left(\left(q^{i-1}-c q^{j-1}\right) \frac{(a q ; q)_{i+j-2}}{\left(a b q^{2} ; q\right)_{i+j-2}}\right)_{1 \leq i, j \leq n}
$$

and apply (4.1) to this determinant. Then we obtain

$$
\begin{gathered}
D_{n}(a, b, c ; q) D_{n-2}\left(a q^{2}, b, c ; q\right)=\frac{q(a q ; q)_{2}}{\left(a b q^{2} ; q\right)_{2}} \cdot D_{n-1}(a, b, c ; q) D_{n-1}\left(a q^{2}, b, c ; q\right) \\
-\frac{q(1-a q)^{n}\left(1-a b q^{3}\right)^{n-2}}{\left(1-a q^{2}\right)^{n-2}\left(1-a b q^{2}\right)^{n}} \cdot D_{n-1}(a q, b, c q ; q) D_{n-1}\left(a q, b, c q^{-1} ; q\right) .
\end{gathered}
$$

Hence we can substitute 1.16 into 4.2 , then replacing $a^{\frac{1}{2}} c^{\frac{1}{2}} q^{\frac{1}{2}} \imath,-a^{\frac{1}{2}} c^{-\frac{1}{2}} q^{\frac{1}{2}} \imath$ and $b^{\frac{1}{2}} \imath$ by $a, b$ and $c$, respectively, we obtain the following corollary.

Corollary 4.1 Let $n$ be a positive integer and $a, b, c$ and $q$ parameters. Then we have

$$
\begin{aligned}
& a b\left(1-q^{n-1}\right)\left(1+c^{2} q^{n-2}\right) p_{n}(0 ; a, b, c,-c ; q) p_{n-2}(0 ; a q, b q, c,-c ; q) \\
= & \left(1-a b q^{n-1}\right)\left(1+a b c^{2} q^{n-1}\right) p_{n-1}(0 ; a, b, c,-c ; q) p_{n-1}(0 ; a q, b q, c,-c ; q) \\
& -(1-a b)\left(1+a b c^{2} q^{2 n-2}\right) p_{n-1}(0 ; a q, b, c,-c ; q) p_{n-1}(0 ; a, b q, c,-c ; q) .
\end{aligned}
$$

Here we derive Corollary 4.1 as a corollary of Theorem 1.1

In fact a more general formula holds. Recently one of the authors has proven that the following quadratic equation in a different method. 
Theorem 4.2 For $r \geq 1$, there holds

$$
\begin{aligned}
& \left(1-a_{0}^{-1}\right)\left(a_{1}-b_{1}\right)_{r+1} \phi_{r}\left[\begin{array}{c}
a_{0} / q, a_{1}, a_{2}, \ldots, a_{r} \\
b_{1} / q, b_{2}, \ldots, b_{r}
\end{array} ; q, z\right]_{r+1} \phi_{r}\left[\begin{array}{c}
a_{0} q, a_{1}, a_{2} q \ldots, a_{r} q \\
b_{1} q, b_{2} q, \ldots, b_{r} q
\end{array} ; q, z\right] \\
& =\left(1-a_{1} / a_{0}\right)\left(1-b_{1}\right)_{r+1} \phi_{r}\left[\begin{array}{c}
a_{0}, a_{1}, a_{2}, \ldots, a_{r} \\
b_{1}, b_{2}, \ldots, b_{r}
\end{array} ; q, z\right]_{r+1} \phi_{r}\left[\begin{array}{c}
a_{0}, a_{1}, a_{2} q \ldots, a_{r} q \\
b_{1}, b_{2} q, \ldots, b_{r} q
\end{array} ; q, z\right] \\
& -\left(1-a_{1}\right)\left(1-b_{1} / a_{0}\right)_{r+1} \phi_{r}\left[\begin{array}{c}
a_{0}, a_{1} / q, a_{2}, \ldots, a_{r} \\
b_{1} / q, b_{2}, \ldots, b_{r}
\end{array} ;, z\right]_{r+1} \phi_{r}\left[\begin{array}{c}
a_{0}, a_{1} q, a_{2} q \ldots, a_{r} q \\
b_{1} q, b_{2} q, \ldots, b_{r} q
\end{array} ; q, z\right)(4.4)
\end{aligned}
$$

This formula gives a simple proof of Theorem 1.1 using the Desnanot-Jacobi adjoint matrix theorem 4.1. But, note that Theorem 2.1 is more general and cannot be derived from the quadratic equation. This thorem may also hint us there could exist a more general formula than Theorem 1.1. But it is not an easy task to find the appropriate entry of the determinant which gives this quadratic relation.

\section{Acknowledgements}

We are grateful to Professor Christian Krattenthaler for his encouragement at the initial stage of this project.

A referee suggested us that Theorem 1.1 can be proven by another method, i.e., using Wilson's result [16] on the Gram determinants for the Askey-Wilson polynomials. As our second proof, this third proof can also avoid our lengthy proof of Theorem 2.1. which is used in our first proof in [6]. But analyzing the referre's suggestion we've discovered Theorem 2.1 type formula not only for the Gram determinants for the Askey-Wilson polynomials but also for the biorthogonal rational function appearing in the paper [16]. This means that we can choose arbitrary rows of the most general Gram determinants in [16] and give an explicit formula. Further we've found that the proof can be much simplified from our original proof in [6] by analizing the referee's idea. Hence our new result will generalize [16], but we don't have enough space to state it here. So we will present our new version in our poster of FPSAC 2013. We slso would like to express our appreciation to the referee for giving us a hint for our new progresses.

\section{References}

[1] D. M. Bressoud. Proofs and Confirmations: The Story of the Alternating-Sign Matrix Conjecture. Cambridge University Press, Cambridge, first edition, 1999. ISBN: 0-521-66170-6.

[2] M. Ciucu and C. Krattenthaler. The interaction of a gap with a free boundary in a two dimensional dimer system. Comm. Math. Phys., 302:253-289, 2011.

[3] G. Gasper and M. Rahman. Basic Hypergeometric Series. Cambridge Univ. Press, Cambridge, second edition, 2004. ISBN: 9-780-52183357-8.

[4] M. Ishikawa, H. Tagawa, and J. Zeng. A $q$-analogue of catalan hankel determinants. RIMS Kôkyûroku Bessatsu, B11:19-42, 2009. available at arXiv:1009.2004.

[5] M. Ishikawa, H. Tagawa, and J. Zeng. Pfaffian decomposition and a pfaffian analogue of $q$-catalan hankel determinants. available at arXiv:1011.5941, 2010.

[6] M. Ishikawa, H. Tagawa, and J. Zeng. A generalization of the mehta-wang determinant and askeywilson polynomials. available at arXiv:1210.5305, 2012. 
[7] R. Koekoek, P. A. Lesky, and R. F. Swarttouw. Hypergeometric Orthogonal Polynomials and Their q-Analogues. Springer-Verlag, Berlin-New York, first edition, 2010. ISBN: 978-3-642-05014-5.

[8] R. Koekoek and R. F. Swarttouw. The askey-scheme of hypergeometric orthogonal polynomials and its $q$-analogue. Technical Report 98-17, Department of Technical Mathematics and Informations, Faculty of Information Technology and Systems, Deift University of Technology, 1998. available at arXiv: 9602214

[9] C. Krattenthaler. A systematic list of two- and three-term contiguous relations for basic hypergeometric series. available at http://www.mat.univie.ac.at/ kratt/artikel/contrel.html, 1993.

[10] C. Krattenthaler. Determinants of (generalized) catalan numbers. J. Statist. Plann. Inference, 140:2260-2270, 2010. available at arXiv:0709.3044.

[11] A. Lascoux. Pfaffians and representations of the symmetric groups. Acta Math. Sin., 25:1929-1950, 2009.

[12] A. Lascoux. Hankel pfaffians, discriminants and kazhdan-lusztig bases. available at http://phalanstere.univ-mlv.fr/ al/ARTICLES/PfaffHankel.pdf, 2012.

[13] M. L. Mehta and R. Wang. Calculation of a certain determinant. Commun. Math. Phys., 214:227$232,2000$.

[14] M. Nishizawa. Evaluation of a certain q-determinan. Linear Algebra Appl., 343:107-115, 2002.

[15] X. G. Viennot. A combinatorial theory for general orthogonal polynomials with extensions and applications. Lecture Notes in Mathematics, 1171:139-157, 1985.

[16] J. A. Wilson. Orthogonal functions from gram determinants. SIAM J. Math. Anal., 22:1147-1155, 1991. 